\title{
ANTONY TROMBA
}

\section{The Euler characteristic of vector fields on Banach manifolds and the problem of Plateau}

Mémoires de la S. M. F., tome 46 (1976), p. 83-89

<http://www.numdam.org/item?id=MSMF_1976_46_83_0>

(C) Mémoires de la S. M. F., 1976, tous droits réservés.

L'accès aux archives de la revue « Mémoires de la S. M. F. » (http://smf. emath.fr/Publications/Memoires/Presentation.html) implique l'accord avec les conditions générales d'utilisation (http://www.numdam.org/conditions). Toute utilisation commerciale ou impression systématique est constitutive d'une infraction pénale. Toute copie ou impression de ce fichier doit contenir la présente mention de copyright.

\section{NumDam}


Journées Géom. dimens. infinie [1975 - LYON]

Bu11. Soc. math. France,

Mémoire 46, 1976, p. $83-89$.

THE EULER CHARACTERISTIC OF VECTOR FIELDS

ON BANACH MANIFOLDS AND THE PROBLEM OF PLATEAU

par Antony TROMBA

In this note we outline a theory of characteristics for vector fields on Banach manifolds, and then give an application to simply connected minimal surfaces. The theory provides a framework to study the question of how the number of simply connected minimal surfaces spanning a simple closed curve $\Gamma \subset R^{3}$ changes as the curve changes.

\section{THE THEORY.}

Let $M$ be a smooth Banach manifold and $K: T^{2} M \rightarrow T M$ a connection map [3]. In [13] the author defines a smooth vector field $X: M \rightarrow T M$ to be Fredholm with respect to $K$ if for each $p \in M$ the covariant derivative of $X$ with respect to $K, \nabla X(p)$, which is a linear map of $\mathrm{T}_{\mathrm{p}} \mathrm{M}$ to itself is 1inear Fredholm [9]. By the index of $\mathrm{X}$ we mean the dim ker $\nabla X(p)$ - dim coker $\nabla X(p)$. If $M$ is connected this index does not depend on $\mathrm{p}$. If $\mathrm{M}$ est not connected, the index is constant on components and we shall require it to be the same for all components. A Fredholm vector field is Palais-Smale if $\nabla X(p)$ is of the form $I+C$, where $C$ is a compact linear map. Palais-Smale vector fields have index zero.

Let $X$ be a Palais-Smale vector field on $M$ with finitely many isolated zeros in the interior of $M$. Then using the degree theory developed in [4] one can define the degree of $X$ at a zero $p$, which we denote by $(\operatorname{deg} X)(p)$. The Euler characteristic $x(X)$ is defined to be

$$
x(X)=\underset{p \in \operatorname{zeros}(X)}{\Sigma}(\operatorname{deg} X)(p) .
$$

If $X$ has no zeros, then $x(X)=0$. By using elementary transversality techniques the Euler characteristic can be defined for Palais-Smale vector fields with a compact set of zeros in the interior of $M$.

DEFINITION. - Suppose $\mathrm{M}$ is a smooth Banach manifold with $\mathrm{X}: \mathrm{M} \rightarrow \mathrm{TM}$ a smooth vector field. A zero $\mathrm{m} \in \mathrm{M}$ is non-degenerate if the Frechet derivative $\mathrm{X}_{*}(\mathrm{~m}): \mathrm{T}_{\mathrm{m}} \mathrm{M}$ is an isomorphism. (At a zero the Frechet derivative agrees with the covariant derivative of $\mathrm{X}$ with respect to any connection on $\mathrm{M})$.

\footnotetext{
*Research partially supported by National Science Foundation grants GP39060 and
MPS72-05055-A02.
} 
As a trivial consequence of this definition it follows that non-degenerate zeros are isolated.

Recall form [4] that the Lie subgroup $\mathrm{GL}_{c}(\mathrm{E})$ of the general linear group GL(E) for a Banach space $E$ is defined as the set of invertible linear maps on $E$ of the form $\mathrm{I}+\mathrm{C}, \mathrm{C}$ compact. In [4] it is shown that $\mathrm{GL}_{\mathrm{C}}(\mathrm{E})$ has two components $\mathrm{GL}_{\mathrm{C}}^{+}(\mathrm{E})$ and $\mathrm{GL}_{\mathrm{C}}^{-}(\mathrm{E})$. Using these ideas we can give a particularly simple description of the Euler-characteristic of a vector field $\mathrm{X}$ with non-degenerate zeros. At a zero $\mathrm{p}$ of $\mathrm{X}$, define

$$
\operatorname{sgn} \nabla X(p)=\left\{\begin{array}{lll}
+1 & \text { if } & \nabla X(p) \in G L_{c}^{+}\left(T_{p} M\right) \\
-1 & \text { if } & \nabla X(p) \in G L_{c}^{-}\left(T_{p} M\right) .
\end{array}\right.
$$

Then

$$
x(X)=\sum_{p \in \operatorname{zeros}(X)}^{\Sigma} \operatorname{sgn} \nabla X(p)
$$

DEFINITION. - Let $\pi_{0}: M \rightarrow[0,1]$ be a smooth fibre bundle over the unit interval with $\pi_{0}^{-1}(t)=M_{t}$ a smooth Banach manifold. Suppose there is a family of maps $\mathrm{k}_{\mathrm{t}}$ with each $\mathrm{K}_{\mathrm{t}}$ a connection for $\mathscr{M}_{\mathrm{t}}$. Two smooth Palais-Smale vector fields $\mathrm{x}_{\mathrm{o}}: \mathscr{M}_{\mathrm{o}} \rightarrow \mathrm{T} \mathscr{M}_{\mathrm{o}}$ and $\mathrm{x}_{1}: \mathrm{M}_{1} \rightarrow \mathrm{TM}_{1}$ are equivalent $\left(\mathrm{x}_{\mathrm{o}} \sim \mathrm{x}_{1}\right)$ if there is a smooth vector field X : $\mathscr{M} \rightarrow \mathrm{T}$ such that

i) $\mathrm{x}_{\mathrm{t}}=\mathrm{x} \mid \mathscr{M}_{\mathrm{t}}: \mathscr{M}_{\mathrm{t}} \rightarrow \mathrm{T} \mathscr{M}_{\mathrm{t}}$, i.e., $\mathrm{x}$ is a vertical family on $\mathscr{M}$;

ii) $\mathrm{x}_{\mathrm{t}}$ is Palais-Smale w.r.t. $\mathrm{k}_{\mathrm{t}}$;

iii) the zeros of $\mathrm{x}$ are compact in the interior of $\mathscr{M}$.

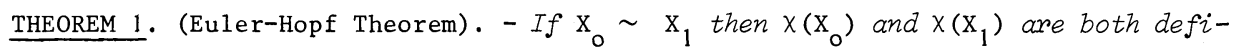
ned and equal.

Proof (A Sketch). - Using transversality theorems which go back to Thom and Abraham we can assume

(a) $\mathrm{X}_{0}$ and $\mathrm{X}_{1}$ have non-degenerate zeros ;

and

(b) $X$ is transverse to the zero section of the vertical subbundle

$$
\mathrm{v}(\mathrm{T} \mathscr{M})=\underset{\mathrm{m} \in \mathrm{M}}{\cup} \mathrm{T} \mathscr{M}_{\mathrm{t}} \text {. }
$$

Conditions (a) and (b) imply that $\mathrm{X}(\mathscr{M}) \cap \mathscr{M}$ is a compact one-dimensional submanifold of the interior of $\mathscr{M}$ with boundary the union of the zeros of $\mathrm{x}_{0}$ and the zeros of $\mathrm{x}_{1}$. Then $\mathrm{x}(\mathscr{M}) \cap \mathscr{M}$ establishes a cobordism between the set $\left\{\mathrm{p}_{1}, \ldots, \mathrm{p}_{\mathrm{k}}\right\}$ and $\left\{q_{1}, \ldots, q_{m}\right\}$. One then shows that if $p_{i}$ and $p_{j}\left(\operatorname{similarly~} q_{i}\right.$ and $\left.q_{j}\right)$ bound the same component of $\mathrm{X}(\mathscr{M}) \cap \mathscr{M}$, then $\operatorname{sgn} \nabla \mathrm{X}\left(\mathrm{p}_{\mathrm{i}}\right)=-\operatorname{sgn} \nabla \mathrm{X}\left(\mathrm{p}_{\mathrm{j}}\right)$ (similarly 
$\left.\nabla X\left(q_{i}\right)=-\operatorname{sgn} \nabla X\left(q_{j}\right)\right)$. If $p_{i}$ and $q_{j}$ bound the same component, then sgn $\nabla X\left(p_{i}\right)=\operatorname{sgn} \nabla X\left(q_{j}\right)$. Using these facts along with formula (1) and pairing off the $\left\{p_{i}\right\}$ and the $\left\{q_{j}\right\}$ we see immediately that $x\left(x_{0}\right)=x\left(x_{1}\right)$.

By applying the Morse theory as developed in [14], [17], one can show that if $X$ and $\mathrm{Y}$ are two vector fields on a manifold $\mathrm{M}$, with $\partial \mathrm{M}=\phi, \mathrm{X} \sim \mathrm{Y}, \mathrm{Y}$ a gradient-like vector field, then the Euler characteristic of $M$ is defined and $X(X)=\chi(M)$. If $\partial \mathrm{M} \neq \phi$ and both $\mathrm{X}$ and $\mathrm{Y}$ point outward along $\partial \mathrm{M}$ the same result holds.

There are conditions which insure that the zeros of a family of vector fields parametrized by some manifold will be generically isolated.

Let $\mathscr{A}$ be a smooth Hilbert manifold, $\pi: \mathscr{M} \rightarrow \mathscr{A}$ a smooth Banach Fibre bundle over $\mathscr{A}$ (the fibre $\pi^{-1}(\mathrm{a})=\mathscr{M}^{\mathrm{a}}$ is a smooth Banach manifold). Let $\mathrm{X}: \mathscr{M} \rightarrow \mathrm{TM}$ be a smooth vector field such that $\mathrm{x}^{\mathrm{a}}=\mathrm{x} \mid \mathscr{M}^{\mathrm{a}}$ is a Fredholm vector field on $\mathscr{M}^{\mathrm{a}}$ with respect to a connection $\mathrm{K}_{\mathrm{a}}$ where $\left\{\mathrm{K}_{\mathrm{a}}\right\}_{\mathrm{a}} \in \mathscr{A}$ is a family of connections. We shall say that such an $\mathrm{X}$ represents a vertical family of Fredholm vector fields, with respect to the family $\left\{\mathrm{K}_{\mathrm{a}}\right\}$.

For each zero $\mathrm{m} \in \mathscr{M}$ of $\mathrm{X}$ the Frechet derivative $\mathrm{X}_{*}(\mathrm{~m})$ can be viewed as a map of $\mathrm{T}_{\mathrm{m}} \mathscr{M}$ into itself. It follows that if $\pi(\mathrm{m})=\mathrm{a}$, then $\mathrm{x}_{*}(\mathrm{~m}) \in \mathrm{T}_{\mathrm{m}} \mathscr{M}^{a}$.

We shall say that a vertical family of Fredholm vector fields $\mathrm{X}$ is vertically transverse to the zero section of $\mathrm{T} \mathscr{M}$ if $\mathrm{x}_{*}(\mathrm{~m}): \mathrm{T}_{\mathrm{m}} \mathscr{M} \rightarrow \mathrm{T}_{\mathrm{m}} \mathscr{M}^{\mathrm{a}}$ is surjective for each zero $\mathrm{m}$ of $\mathrm{x}$. In this case, we write $\mathrm{x} \prod_{\mathrm{v}} \mathrm{z}(\mathrm{T} \mathscr{M})$. We can interpret this geometrically as meaning that $\mathrm{X}(\mathscr{M})$ is transverse to the zero section of the vertical subbundle of $\mathrm{T} \mathscr{M}$.

Assume now that $\mathrm{Y}$ is a vector field on a Banach Finsler manifold $M$. Therefore we have a norm \|\|$_{m}: T_{m} M \rightarrow R$ on each fibre of $T M$ which varies continuously over $M$ [e.g., see [13]]. We shall say that a vector field $Y$ on a Banach Finsler manifold

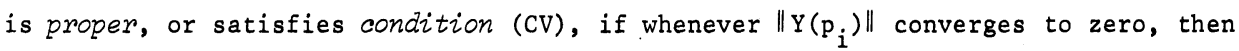
$\left\{\mathrm{p}_{\mathrm{i}}\right\}$ has a convergent subsequence.

Suppose $\pi: \mathscr{M} \rightarrow \mathscr{A}$ is a fibre bundle as above with $\|: \mathrm{v}(\mathrm{T} . \mathscr{U}) \rightarrow \mathrm{R}$ is a Finsler on the vertical subbundle of $\mathrm{T} . \mathcal{M}$. A vertical family $\mathrm{X}$ is proper if whenever $\left\|\mathrm{X}\left(\mathrm{p}_{i}\right)\right\|$ converges to zero and $\pi\left(p_{i}\right)$ converges, then $\left\{p_{i}\right\}$ has a convergent subsequence.

THEOREM 2. - Suppose $\mathrm{x} 巾_{\mathrm{v}} \mathrm{z}(\mathrm{T}, \mathscr{M})$, where $\mathrm{x}$ represents a proper vertical family of index zero vector fields. Then there exists an open dense set $\boldsymbol{V} \subset \mathscr{A}$ with the property that for a $\in \mathscr{V}, \mathrm{x}^{\mathrm{a}}: \mathscr{M}^{\mathrm{a}} \rightarrow \mathrm{T} \cdot \mathcal{M}^{\mathrm{a}}$ has only non-degenerate zeros. 


\section{APPLICATIONS TO THE PLATEAU PROBLEM.}

Since its formulation by PLATEAU in the 19th century, 1ittle (see [2], [10]) has been known about the number of simply connected minimal surfaces spanning a simple closed curve $\Gamma \subset \mathrm{R}^{3}$. Existence was proved in the thirties by J. DOUGLAS [1] and T. RADO [11], and most contemporary research has been directed toward regularity results for such minimal surfaces.

Examples of $\Gamma$ are known which seem to indicate that it is possible to have as many simply connected minimal surfaces spanning a curve (including infinitely many) as one would like. Nothing was known about how the number changed as the curve. changed. In the paragraphs below we state theorems which partially answer these questions in terms of our topological theory. They follow from modified versions of Theorem 1 and 2 in Section 1 .

Let $\mathrm{H}^{\mathrm{r}+2}\left(\mathrm{~S}^{1}, \mathrm{R}^{\mathrm{n}}\right)$ be the Sobelev Hilbert space of $\mathrm{H}^{\mathrm{r}+2}$ maps of the unit circle $\mathrm{S}^{1}$ into $\mathrm{R}^{\mathrm{n}}$, with $\mathrm{r} \geqslant 5$. Let $\mathscr{A}=\operatorname{Emb}\left(\mathrm{S}^{1}, \mathrm{R}^{3}\right)$ be the open submanifold of $\mathrm{H}^{\mathrm{r}+2}\left(\mathrm{~S}^{1}, \mathrm{R}^{3}\right)$ which consists of embeddings of $\mathrm{S}^{1}$ into $\mathrm{R}^{3}$. Let $\Gamma$ be the image of such an embedding $\alpha \in \mathscr{A}$. Set $\eta^{\alpha}$ to be the component of $\mathrm{H}^{2}\left(\mathrm{~S}^{1}, \Gamma\right)$ \{ the $\mathrm{C}^{\mathrm{r}}$ Hilbert manifold of $\mathrm{H}^{2}$ maps from $S^{1}$ to $\Gamma$ \} determined by the embedding $\alpha$. Let $\mathscr{M}^{\alpha}$ be the open submanifold of $\eta^{\alpha}$ consisting of the diffeomorphisms. For every $u \in \mathrm{H}^{2}\left(\mathrm{~S}^{1}, \Gamma\right) \subset \mathrm{H}^{2}\left(\mathrm{~S}^{1}, \mathrm{R}^{3}\right)$ we can extend $u=\left(u_{1}, \ldots, u_{n}\right)$ harmonically to the disc $\mathscr{D}$. Define the smooth Dirichlet energy functional $\mathrm{E}_{\alpha}: n^{\alpha} \rightarrow \mathrm{R}$ by

$$
E_{\alpha}(u)=\frac{1}{2} \sum_{i=1}^{3} \int_{\mathscr{D}}\left|\left(\frac{\partial u_{i}}{\partial x}\right)^{2}+\left(\frac{\partial u_{i}}{\partial y}\right)^{2}\right| d x d y .
$$

We can write the Dirichlet functional as an integral over $S^{1}=\partial \mathscr{D}$ as follows. Let $\theta \rightarrow \frac{\partial u}{\partial r}(\theta)$ represent the partial derivative with respect to the polar coordinate $r$ of the harmonic extension of $u$ to $\mathscr{D}$ evaluated at $r=1$. This agrees with the normal derivative on $S^{1}$ of the harmonic extension. Let $<,>$ denote the $\mathbb{R}^{3}$ inner product. Then applying Green's theorem to (2) we find that

$$
E_{\alpha}(u)=\frac{1}{2} \int_{S}<\frac{\partial u}{\partial r}, u>d \theta .
$$

Denote by $\overline{\mathscr{M}}^{\alpha}$ the closure of $\mathscr{M}^{\alpha}$ in $n^{\alpha}$.

J. DOUGLAS showed in his pioneering work [1] that the harmonic extensions of the critical points of $\mathrm{E}$ in $\overline{\mathscr{M}}^{\alpha}$ are the simply connected minimal surfaces spanning $\Gamma$. For a somewhat simpler proof see [16]. 
THEOREM 3. - There exists a smooth connection $\mathrm{K}_{\alpha}$ on the second tangent bundle $\mathrm{T}^{2} \mathrm{n}^{\alpha}$ and a smooth vector field $\mathrm{x}^{\alpha}: \mathrm{n}^{\alpha} \rightarrow \mathrm{Tn}^{\alpha}$ which is Palais-Smale with respect to the connection $\mathrm{K}_{\alpha}$ and whose zeros are precisely all the critical points of $\mathrm{E}_{\alpha}$. Moreover,

$$
\mathrm{X}^{\alpha}\left(\mathrm{E}_{\alpha}\right)=\mathrm{dE}_{\alpha}(\mathrm{u})\left(\mathrm{x}^{\alpha}(\mathrm{u})\right) \geqslant 0 .
$$

Proof (A Sketch). - Let $h, k \in \mathrm{T}_{\mathrm{u}} \eta^{\alpha}$. Define the weak Riemannian structure $\ll, \gg: \mathrm{Tn}^{\alpha} \times \mathrm{Tn}^{\alpha} \rightarrow \mathrm{R}$ by setting

$$
\ll \mathrm{h}, \mathrm{k} \gg=\int_{\mathrm{S}^{1}}<\frac{\partial \mathrm{h}}{\partial \mathrm{r}}, \mathrm{k}>\mathrm{d} \theta .
$$

By definition $\eta^{\alpha} \subset H^{2}\left(S^{1}, \mathbb{R}^{3}\right)=H$ is a submanifold. For each $u \in \eta^{\alpha}$

$$
\mathrm{H}=\mathrm{T}_{\mathrm{u}} \mathrm{n}^{\alpha} \oplus\left(\mathrm{T}_{\mathrm{u}} \mathrm{n}^{\alpha}\right)^{\perp}
$$

where $\left(T_{u} n^{\alpha}\right)^{\perp}$ is the weak orthogonal complement of $T_{u} n^{\alpha}$ in $H$. In a standard way, one can use the splitting (3) to define the connection $\mathrm{K}_{\alpha}$ on $n^{\alpha}$.

The vector field $\mathrm{X}^{\alpha}$ is the "gradient" of $\mathrm{E}_{\alpha}$ with respect to $\ll, \gg$. It is necessary to check that this weak gradient gives a smooth vector field on $\eta^{\alpha}$ and we use regularity theorems from the theory of elliptic partial differential equations to accomplish this. The connection $K_{\alpha}$ and the vector field $x^{\alpha}$ are both defined natural$1 y$ in terms of the weak Riemannian structure. By a direct computation we show that $\mathrm{X}^{\alpha}$ is Palais-Smale with respect to $\mathrm{K}_{\alpha}$.

DEFINITION. - Let $\mathrm{u} \in \mathrm{n}^{\alpha}$ be a minimal surface. A branch point $\mathrm{p} \in \mathscr{D}$ of $\mathrm{u}$ is a point where the map u : $\mathscr{D} \rightarrow \mathbb{R}^{3}$ fails to be an immersion. An embedding $\alpha \in \mathscr{A}$ is fine if all minimal surfaces spanning $\Gamma=\alpha\left(S^{1}\right)$ are free of branch points.

In [10] RADO showed that if $\alpha$ is not "too complicated", then $\alpha$ is fine. In particular, he showed that $\alpha$ is fine if there existed no point $q \in \mathbb{R}^{3}$ such that every hyperplane through $q$ intersected $\Gamma$ in at least four points.

THEOREM 4. - Let I $\subset \mathscr{A}$ be the set of fine embeddings. Then I Is open in $\mathscr{A}$, and hence open in $\mathrm{H}^{\mathrm{r}+2}\left(\mathrm{~S}^{1}, \mathbb{R}^{3}\right)$.

Conjecture. - I is dense in $\mathscr{A}$, or perhaps the open set of curves which admit only minimal surfaces without boundary branch points is dense in $\mathscr{A}$.

Let $G$ be the three dimensional non-compact lie group of bijective holomorphic maps of the disc onto itself. The functional $\mathrm{E}_{\alpha}$ and the vector field $\mathrm{x}^{\alpha}$ of Theorem 3 will be equivariant with respect to the action of $G$. Therefore, there is no hope that the critical points of $\mathrm{E}_{\alpha}$ in $\bar{M}^{\alpha}$ will be isolated or non-degenerate in the 
sense described earlier since the orbits of any critical point will consist of critical points. Let $\mathrm{u} \in \overline{\mathscr{M}}^{\alpha}$ be a zero of $\mathrm{x}^{\alpha}$, and let $\mathcal{O}_{\mathrm{G}}$ (u) denote the orbit of under $G$. Then $\mathcal{O}_{G}(u)$ is an immersed three-dimensional submanifold of $\eta^{\alpha}$ consisting of critical points of $E_{\alpha}$. We call $\mathcal{O}_{G}(u)$ a critical submanifold of $n^{\alpha}$. The covariant derivative of $\mathrm{x}^{\alpha}$ at $\mathrm{u}$ induces a homomorphism of the normal bundle of $\mathcal{O}_{\mathrm{G}}(\mathrm{u})$ into itself. A critical point $u$, or more precisely $\mathcal{O}_{G}(u)$, is non-degenerate if this induced homomorphism is an isomorphism.

Applying a modified version of Theorem 2 and some regularity theorems of HILDEBRANDT [5] and NITSCHE [7] and others we obtain

THEOREM 5. - For an open dense set of embeddings $\mathscr{V} \subset \mathscr{I}$ the zeros of $\mathrm{x}^{\alpha}, \alpha \in \mathscr{V}$, in $\mathscr{M}^{\alpha}$ are non-degenerate (and therefore isolated) three-dimensional submanifolds of $n^{\alpha}$. Moreover, for such $\alpha \in \mathscr{V}$ there are only finitely many such critical submanifolds.

In general minimal surfaces on the same orbit are identified. Doing this we obtain THEOREM 6. - If $\alpha \in \mathscr{V}$ and $\gamma \in \mathscr{V}, \gamma=\alpha+\rho$, is sufficiently close to $\alpha$, then the minimal surfaces spanning $\gamma$ are smooth functions of the parameter $\rho$.

COROLLARY. - If $\alpha \in \mathscr{V}$ and $\gamma \in \mathscr{V}$ is sufficiently close to a then the geometric number of minimal surfaces spanning $\gamma$ is equal to the number spanning $\alpha$.

Let $\Gamma=\alpha\left(S^{1}\right), \alpha \in \mathscr{A}$, be a plane curve. The Riemann-Osgood mapping theorem implies that there is a unique minimal surface'spanning $\Gamma$. This will be the conformal map of the disc onto the bounded component of $\mathrm{C}$ determined by $\Gamma$.

THEOREM 7. - If $\Gamma$ is a plane curve, then the unique minimal surface which spans it is non-degenerate.

COROLLARY. - Any curve sufficiently close to a plane curve has a unique minimal surface spanning it.

Let $\gamma$ belong to $\mathscr{I}$. We can define the Euler characteristic of the corresponding vector field $x^{\alpha}$, and we take this to be the definition of the algebraic number of minimal surfaces spanning the image $\gamma\left(S^{1}\right)$.

We conclude with an application of a modified version of Theorem 1 .

THEOREM 8. - Let $\gamma_{0}$ and $\gamma_{1}$ be fine embeddings. Suppose further that $\gamma_{0}$ is isotopic to $\gamma_{1}$ through a family $\gamma_{t}, 0 \leqslant t \leqslant 1$ of fine embeddings. Then the algebraic number surfaces spanning $\gamma_{0}$ is equal to the number spanning $\gamma_{1}$. 
REFERENCES

[1] DOUGLAS J., Solution to the problem of Plateau, Trans. Amer. Math. Soc., 33, (1931), 263-321.

[2] COURANT R., Dimichlet's Principle, Interscience, New-York, 1950.

[3] ELIASSON H., Geometry of manifolds of mops, J. Diff. Geometry, 1 (1967), 169-194.

[4] ELWORTHY K.K., and TROMBA A.J., Differentiable structures and Fredholm maps on Banach manifolds, Proc. Symp. on Global Analysis, Berkeley, 1968.

[5] HILDEBRANDT S., Boundary behavior of minimal surfaces, Arch. Rat. Mich. Ana1.

[6] MORSE M., and TOMPKINS C., Existence of minimal surfaces of general critical type, Proc. Nat. Acad. Sciences U.S.A., 25 (1939), 153-158.

[7] NITSCHE J.C.C., The boundary behavior of minimal surfaces, Kellog's theorem and branch points on the boundary, Inventiones Math., 8 (1.969), 313-333.

[8 ] OSSERMAN R., A survey of Minimal Surfaces, Van Nostrand, Princeton, 1969.

[9] PALAIS R., Lectures on the Atiyah-Singer Index Theorem, Princeton, 1964.

[10] RADO T., On the problem of Plateau, Ergebnisse der Mathematik und Ihrer Grenzgebeite, Springer-Verlag, Berlin, 1933.

[11] RADO T., The problem of least area and the problem of Plateau, Math. Z., 32 (1930), 763-796.

[12] SCHIfFMAN M., Plateau's problem for non-relative minima, Ann. Math., 40 (1939), 834-854.

[13] TROMBA A., Fredholm vector fiezds and a transversality theorem, preprint.

[14] TROMBA A., A generalized opproach to Morse theory, to appear in J. Diff. Geometry.

[15] TROMBA A., The Morse-Sard-Brown theorem and the problem of Plateau, to appear in the Amer. J. Math.

[16] TROMBA A., On the number of simply connected minimal surfaces spanning a curve in $\mathrm{R}^{3}$, in preparation.

[17] TRомBA A., Théorie de Morse sur les variétés de Banach, C.R. Acad. Sc. Paris, (1975).

\author{
Antony TROMBA \\ The Institute for Advanced Study \\ Princeton, N.J. 08540 \\ and \\ The University of California at Santa Cruz \\ ๙ぇぇぇぇ
}

